\title{
Extension Error Set Based on Extension Set
}

\author{
Qixin Ye, Jinge Zhou \\ Guangdong University of Technology, School of Management, Guangzhou, China \\ Email: 411682485@qq.com
}

Received July 31, 2013; revised August 31, 2013; accepted September 7, 2013

Copyright (c) 2013 Qixin Ye, Jinge Zhou. This is an open access article distributed under the Creative Commons Attribution License, which permits unrestricted use, distribution, and reproduction in any medium, provided the original work is properly cited.

\begin{abstract}
This paper gives the concepts of extension error set and fuzzy extension error set, discusses diverse extension error set and fuzzy extension error set based on extension set and error set, and puts forward the relevant propositions and operations. Finally, it provides proofs of the soundness and completeness for the propositions and operations.
\end{abstract}

Keywords: Extension Set; Error Set; Fuzzy Extension Error Set

\section{Introduction}

In the field of fuzzy mathematics, the research of set mainly concentrates on the static form of fuzzy set and its effective forms of reasoning and rule. However, the dynamic changes of the fuzzy set are important parts of set research. In this paper, firstly, we study extension error set and fuzzy extension error set's dynamic concept based on the theory of error eliminating and extenics. Then, we research diverse extension error set and fuzzy extension error set, and put forward the relevant propositions and operations. Finally, we provide proofs of the soundness and completeness for the propositions and operations. In one word, because of the study of extension error set, this paper has very important theoretical and practical significance in different fields.

\section{Basic Definitions}

\subsection{Matter-Element [1-6]}

Definition 2.1.1 An ordered triple composed of the measure $v_{m}$ of $O_{m}$ about $c_{m}$, with matter $O_{m}$ as object, and cm as characteristic

$$
M=\left(O_{m}, c_{m}, v_{m}\right)
$$

As the fundamental element for matter description, it's referred to as 1-dimensional matter-element, and $O_{m}$, vm are referred to as the three key elements of matterelement $M$, within which, the two-tuples composed of $\mathrm{cm}$ and $v_{m}\left(c_{m}, v_{m}\right)$ is referred to as the characteristic-element of matter $O_{m}$.

For convenience, the whole matter-element is ex- pressed as $£(M)$, the whole matter is expressed as $£\left(O_{m}\right)$, and whole characteristic as $£\left(c_{m}\right)$. The domain of measure of characteristic $c_{m}$ is expressed as $V\left(c_{m}\right)$, referred to as the domain of measure of $c_{m}$.

A matter with multiple characteristics, similar to 1dimensional matter-element, can be defined as a multidimensional matter-element:

Definition 2.1.2 The array composed of matter $O_{m}$, $n$-names of characteristics of $c_{m 1}, c_{m 2}, \cdots, c_{m n}$ and the corresponding measure $v_{m i}(i=1,2, \cdots, n)$ of $O_{m}$ about $c_{m i}(i=1,2, \cdots, n)$

$$
M=\left[\begin{array}{ccc}
O_{m} & c_{m 1} & v_{m 1} \\
& c_{m 2} & v_{m 2} \\
\vdots & \vdots \\
& c_{m n} & v_{m n}
\end{array}\right]=\left(O_{m}, C_{m}, V_{m}\right)
$$

is referred to as $n$-dimensional matter-element, wherein

$$
C_{m}=\left[\begin{array}{c}
C_{m 1} \\
C_{m 2} \\
\vdots \\
C_{m n}
\end{array}\right], V_{m}=\left[\begin{array}{c}
v_{m 1} \\
v_{m 2} \\
\vdots \\
v_{m n}
\end{array}\right] \text {. }
$$

\subsection{Affair-Element}

Interaction between matters is referred to as affair, described by affair-element.

Definition 2.2.1 The ordered triple composed of action $O_{a}$, action's characteristic $c_{a}$ and the obtained measure $v_{a}$ of $O_{a}$ about $c_{a}$

$$
A=\left(O_{a}, C_{a}, v_{a}\right)
$$


is used as the fundamental element for affair description, referred to as 1-dimensional affair-element.

Basic characteristics of action include dominating object, acting object, receiving object, time, location, degree, mode, and tool, etc.

Definition 2.2.2 The array composed of action $O_{a}$, $n$-characteristics $c_{a 1}, c_{a 2}, \cdots, c_{a n}$ and the obtained measure $v_{a 1}, v_{a 2}, \cdots, v_{a n}$, of $O_{a}$ about $c_{a 1}, c_{a 2}, \cdots, c_{a n}$

$$
\left[\begin{array}{ccc}
O_{a} & c_{a 1} & v_{a 1} \\
& c_{a 2} & v_{a 2} \\
& \vdots & \vdots \\
& c_{a n} & v_{a n}
\end{array}\right]=\left(O_{a}, C_{a}, V_{a}\right)^{\stackrel{\Delta}{=} A}
$$

is referred to as $n$-dimensional affair-element, wherein

$$
C_{a}=\left[\begin{array}{c}
c_{a 1} \\
c_{a 2} \\
\vdots \\
c_{a n}
\end{array}\right], \quad V_{a}=\left[\begin{array}{c}
v_{a 1} \\
v_{a 2} \\
\vdots \\
v_{a n}
\end{array}\right]
$$

\subsection{Relation-Element}

In the boundless universe, there is a network of relations among any matter, affair, person, information, knowledge and other matter, affair, person, information and knowledge. Because of interaction and interplay among these relations, the matter-element, affair-element and relation-element describing them also have various relations with other matter-elements, affair-elements and relation-elements, and the changes of theses relations will also be interacting and interplaying. Relation-element is a formalized tool to describe this kind of phenomena.

\section{The Research of Extension Error Set}

We research extension error set based on the theory of Extenics, and explore classical extension error set, fuzzy extension error set, multivariate extension error set. Moreover, we put forward the relevant propositions and operations. According to thses propositions and operations, we provide some proofs.

\subsection{The Definition of Extension Error Set}

Suppose $U(t)$ is an object set, $S(t)$ is a set of association rules, if

$$
\begin{gathered}
E=\{((U(t), M(t), A(t), R(t)), x(t)=f(S \neq>u(t))) \mid \\
(U(t), M(t), A(t), R(t))=u(t), \\
f \in U(t), r, x(t)=f(S \neq>u(t))\}
\end{gathered},
$$

we call that " $E$ " is an extension error set for association rule $S(t)$ in domain $U(t)$. In detail, $U$ is a domain,
$S(t)$ (incidence - standard) is a set of association rules, $M$ refers to the matter-element, $A$ representative affairelement, $R$ represents the relationship between relationelement; $X(t)=f(S>u(t))$ represents the correlation functions of extension error set, $R$ is the real number field, $T$ refers to the time. In this paper we take extension error set as a complex system, its' elements as subsystems.

So,

$$
\begin{gathered}
U_{+}=\{u(t) \mid(u(t), x(t)) \in E, x(t)>0\}, \\
U_{-}=\{u(t) \mid(u(t), x(t)) \in E, x(t)<0\}, \\
\tilde{U}=\{u(t) \mid(u(t), x(t)) \in E, x(t)=0\}, \\
U_{+}=\{u(t) \mid(u(t), x(t)) \in E, x(t) \geq 0, \\
\\
T(f(S \neq>u(t)))<0\}, \\
U_{-}=\{u(t) \mid(u(t), x(t)) \in E, x(t) \leq 0, \\
\\
T(f(S \neq>u(t)))>0\}, \\
\tilde{U}=\{u(t) \mid(u(t), x(t)) \in E, T(f(S \neq>u(t)))=0\},
\end{gathered}
$$

are called extension error set's extension of the domain, negative extension field, extension, stable domain and negative stable region, critical region respectively.

$$
\begin{aligned}
& S(t) \neq>u(t) \\
& \text { contain }\left\{\begin{array}{l}
\text { 1) } u(t) \text { and } S(t) \text { contradictious } \\
\text { 2) } S(t) \text { completely can not push out } u(t) \\
\text { 3) } S(t) \text { some part can not push out } u(t) \\
\text { 4) } S(t) \text { possible can not push out } u(t)
\end{array}\right.
\end{aligned}
$$

In the definition $f(S \neq>u(t))$ should be a general situation $f(u(t), S(t))$.

Proposition 3.1.1 In $U$, if $S_{1}=S_{2}, f_{1}=f_{2}$, then

$$
\begin{aligned}
E_{1}=\{( & \left.(U(t), M(t), A(t), R(t)), x(t)=f_{1}(S \neq>u(t))\right) \mid \\
& (U(t), M(t), A(t), R(t))=u(t) \in U(t), \\
& \left.f_{1} \in U(t)^{\prime} r, x(t)=f_{1}(\neq>u(t))\right\} \\
E_{2}=\{( & \left.(U(t), M(t), A(t), R(t)), x(t)=f_{2}(S \neq>u(t))\right) \mid \\
& (U(t), M(t), A(t), R(t))=u(t) \in U(t), \\
& \left.f_{2} \in U(t) r, x(t)=f_{2}(S \neq>u(t))\right\}
\end{aligned}
$$

have $E_{1}=E_{2}$, vice versa.

Proof, when $S_{1}=S_{2}, f_{1}=f_{2}$,

$$
\forall u(t) \in U, x(t)=f_{1}(S \neq>u(t))=f_{2}(S \neq>u(t))=y(t),
$$


so, $\forall u(t) \in U$, when

$(u(t), x(t)) \in E_{1},(u(t), y(t)) \in E_{2}$ have $\quad x(t) \neq y(t)$, so, $E_{1}=E_{2}$, conversely, if $E_{1}=E_{2}$, we can know $S_{1}=S_{2}$. Also if $f_{1} \Rightarrow f_{2}$ Is true in $U$, then $\exists u(t) \in U$, have $f_{1}(S \neq>u(t)) \neq f_{2}(S \neq>u(t))$, so, for

$(u(t), x(t)) \in E_{1},(u(t), y(t)) \in E_{2}$

$(u(t), x(t)) \in E_{1},(u(t), y(t)) \in E_{2}$, have $x(t) \neq y(t)$, This contradiction with $E_{1}=E_{2}$. so, $f_{1}=f_{2}$.

The end.

\subsection{The Class of Extension Error Set}

We according to the features of the elements can be divided:

1) Classic extension error set

$$
\begin{aligned}
E=\{( & (U(t), M(t), A(t), R(t)), x(t)=f(S \neq>u(t))) \mid \\
& (U(t), M(t), A(t), R(t))=u(t) \in U \\
& \left.f \in U^{\prime}\{0,1\}, x(t)=f(S \neq>u(t))\right\}
\end{aligned}
$$

2) Fuzzy extension error set

$$
\begin{gathered}
E=\{((U(t), M(t), A(t), R(t)), x(t)=f(S \neq>u(t))) \mid \\
(U(t), M(t), A(t), R(t))=u(t) \in U \\
\left.f \in U^{\prime}[0,1], x(t)=f(S \neq>u(t))\right\}
\end{gathered}
$$

3) Have critical point extension error set

$$
\begin{gathered}
E=\{((U(t), M(t), A(t), R(t)), x(t)=f(S \neq>u(t))) \mid \\
(U(t), M(t), A(t), R(t))=u(t) \in U \\
f \in U(-\infty,+\infty), x(t)=f(S \neq>u(t))\}
\end{gathered}
$$

\section{The Research of Fuzzy Extension Error Set}

This section mainly research the definition, relation, operation of extension error set.

\subsection{The Definition of Fuzzy Extension Error Set}

Definition 4.1.1 Suppose $U$ is object set, $\mathrm{S}$ is a set of association rules in $U$, if

$\underset{\sim}{E}=\{(u, x) \mid u \in U, x=f(S \neq>u), f \subseteq U[0,1]\}$, we call that $\underset{\sim}{E}$ is a fuzzy extension error set for $S$ in $U$.

\subsection{The Relation between Fuzzy Extension Error Sets}

4.2.1. Equation

Definition 4.2.1 Suppose

$$
\begin{aligned}
& \quad{\underset{\sim}{1}}_{1}=\{(u, x) \mid u \in U, x=f(S \neq>u), f \subseteq U[0,1]\}, \\
& {\underset{\sim}{*}}_{2}=\left\{(u, y) \mid u \in U, y=f_{2}\left(S_{2} \neq>u\right), f_{2} \subseteq U[0,1]\right\}, \text { if } \\
& \forall u \in U \text {, have }(u, x) \in{\underset{\sim}{1}}_{1}, \quad(u, x) \in \underset{\sim}{E_{2}} \text {, make } x=y \\
& \text { and } S_{1}=S_{2} \text {, we call that } \underset{\sim}{E_{1}}=\underset{\sim 2}{E_{2}} \text { for association rule } \\
& S_{1} \text { or } S_{2} .
\end{aligned}
$$

\subsubsection{Subset}

Definition 4.2.2 Suppose $U_{1}, U_{2}$ are subset in $U$, and ${\underset{\sim}{1}}_{1}=\{(u, x) \mid u \in U, x=f(S \neq>u), f \subseteq U[0,1]\}$,

${\underset{\sim}{2}}_{2}=\left\{(u, y) \mid u \in U, y=f_{2}\left(S_{2} \neq>u\right), f_{2} \subseteq U[0,1]\right\}$, if

$U_{1} \subseteq U_{2}$ for association rule $S, \underset{\sim}{E_{1}}$ is the subset of $\underset{\sim}{E_{2}}$, so $\underset{\sim 1}{E_{1}} \subseteq E_{2}$, or ${\underset{\sim}{E}}_{2} \supseteq \underset{\sim}{E_{1}}$.

By definition, there are clearly established the following proposition:

Proposition 4.2.1 Suppose $\underset{\sim}{E_{1}}, \underset{\sim}{E_{2}}, \underset{\sim}{E_{3}}$ are subset for association rule $S$ :

1) $E_{1} \subseteq E_{1}$,

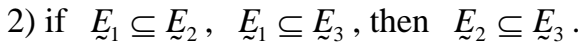

Proposition 4.2.3 Suppose $\underset{\sim 1}{E_{1}}, \underset{\sim}{E_{2}}$ are fuzzy sets for association rule $S_{1}, S_{2}$ :

Then ${\underset{\sim}{1}}_{1}=\{(u, x) \mid u \in U, x=f(S \neq>u), f \subseteq U[0,1]\}$,

$$
{\underset{\sim}{2}}_{2}=\left\{(u, y) \mid u \in U, y=f_{2}\left(S_{2} \neq>u\right), f_{2} \subseteq U[0,1]\right\} \quad \text { If }
$$

$\forall u \in U,(u, x) \in \underset{\sim 1}{E_{1}}, \quad(u, x) \in \underset{\sim}{E_{2}}$, have $x \leq y$, then ${\underset{\sim}{1}}_{1} \leq \underset{\sim 2}{E_{2}}$ or ${\underset{\sim}{\sim}}_{2} \geq \underset{\sim 1}{E_{1}}$ for association rule in $U$.

Proposition 3.1.2.4 Suppose $\underset{\sim 1}{E_{1}}, \underset{\sim}{E_{2}}, E_{\sim}$ are fuzzy subsets for association rule $S_{1}, S_{2}, S_{3}$ in $U$

1) $E_{1} \subseteq \underset{\sim 1}{E_{1}}$,

2) if $\underset{\sim}{E_{1}} \subseteq \underset{\sim 2}{E_{2}}, \quad \underset{\sim 1}{E_{1}} \subseteq \underset{\sim 3}{E_{3}}$, then $\underset{\sim 2}{E_{2}} \subseteq \underset{\sim}{E_{3}}$.

\subsection{The Operations of between Fuzzy Extension Error Sets}

\subsubsection{The Union of Fuzzy Extension Error Set}

If $f\left(x, y, S_{1}, S_{2}\right) \equiv 0$, then the definition of Fuzzy extension error set's union for association rule $S_{1}, S_{2}$ :

Definition 4.3.1.1 Suppose $\underset{\sim}{E_{1}}$ and $\underset{\sim}{E_{2}}$ are fuzzy sets for association rule $S_{1}, S_{2}$ in $U$, and

${\underset{\sim}{3}}_{3}=\left\{(u, z) \mid(u, x) \in E_{\sim},(u, y) \in E_{\sim}, z=\max (x, y)\right\}$, then ${\underset{\sim}{3}}_{3}={\underset{\sim}{1}}_{1} \vee{\underset{\sim}{2}}_{2}$, means union.

Proposition 3.2.2.1 Suppose ${\underset{\sim}{1}}_{1},{\underset{\sim}{2}}_{2}$ are subsets for association rule $S_{1}, S_{2}$, then

1) $E_{1} \vee E_{1}=E_{1}$;

2) $\underset{\sim}{E_{1}} \vee \underset{\sim}{E_{2}}=\underset{\sim}{E_{2}} \vee \underset{\sim}{E_{1}}$;

3) if $\underset{\sim}{E_{1}} \leq \underset{\sim}{E_{2}}$, then $\underset{\sim 1}{E_{1}} \leq \underset{\sim}{E_{1}} \vee \underset{\sim}{E_{2}}=\underset{\sim}{E_{2}}$.

4.3.2. The Intersection of Fuzzy Extension Error Set Definition 4.3.2.1 Suppose $\underset{\sim}{E_{1}}$ and $\underset{\sim}{E_{2}}$ are fuzzy sets for association rule $S_{1}, S_{2}$ in $U$ and

${\underset{\sim}{3}}_{3}=\left\{(u, z) \mid(u, x) \in \underset{\sim}{E_{1}},(u, y) \in \underset{\sim}{E_{2}}, z=\min (x, y)\right\}$, then 
$\underset{\sim}{E_{3}}=\underset{\sim}{E_{1}} \wedge \underset{\sim 2}{E_{2}}$ means intersection.

Proposition 4.3.2.1 Suppose $\underset{\sim}{E_{1}}, \underset{\sim}{E_{2}}, \underset{\sim}{E_{3}}$ are subsets for association rule $S_{1}, S_{2}, S_{3}$ then

1) $\underset{\sim 1}{E_{1}} \wedge E_{1}=E_{1}$

2) $\underset{\sim}{E_{1}} \wedge \sim_{\tilde{E}_{2}}={\underset{\sim}{E}}_{2} \wedge{\underset{\sim}{1}}_{1}$

3) $\underset{\sim}{\tilde{E}_{1}} \wedge\left(\underset{\sim}{\tilde{\sigma}_{2}} \wedge \underset{\sim}{\mathbb{\sim}_{3}}\right)=\left({\underset{\sim}{\sim}}_{1} \wedge{\underset{\sim}{E}}_{2}\right) \wedge{\underset{\sim}{E}}_{3}$

\section{Conclusion}

Extenics and error eliminating theory have increasingly attracted the attention of academia and industry, especially in the fields of management and decision-making. So we study the extension error set and fuzzy extension error set. But, what we have done is not enough. It's in administrative before our theory is perfect. So, we call for more scholars from all over the world to do research about extenics and error eliminating theory. Only in this way, can they have wider value of applications in more fields.

\section{REFERENCES}

[1] W. Cai, C. Y. Yang and W. C. Lin, "Extension Engineering Methods,” Science Press, Beijing, 2003.
[2] C. Y. Yang, G. H. Wang, Y. Li and W. Cai, "Study on Knowledge Reasoning Based on Extended Formulas,” International Conference on AIAI, Springer, New York, Vol. 9, 2005, pp. 797-805.

[3] Y. Q. Yu, Y. Huang and M. H. Wang, "The Related Matter-Elements in Extension Detecting and Application," Proceedings of IEEE International Conference on Information Technology and Applications (ICITA), July 2005, pp. 411-418.

[4] Y. Q. Yu, H. X. Peng and W. Q. Ye, "The Principle of Extension Detecting with Extension Sets," International Conference on Computer, Communication and Control Technologies (CCCT), Florida, July 2003, pp. 113-118.

[5] Z. Chen and Y. Q. Yu, "To Find the Key Matter-Element Research of Extension Detecting," International Conference on Computer, Communication and Control Technologies (CCCT), Florida, July 2003.

[6] Y. Huang, B. Zeng and M. H. Wang, "The Basic MatterElement Research of Extension Detecting,” International Conference on Computer, Communication and Control Technologies (CCCT), Florida, July 2003. 